\title{
A fuzzy tri-level decision making algorithm and its application in supply chain
}

\author{
Zhen Zhang ${ }^{1,2}$, Guangquan Zhang ${ }^{2}$, Jie Lu ${ }^{2}$, Chonghui Guo ${ }^{1}$ \\ ${ }^{1}$ Institute of Systems Engineering, Dalian University of Technology, Dalian 116024, P. R. China \\ ${ }^{2}$ Lab of Decision Systems and e-Service Intelligence, Centre for Quantum Computation and Intelligent \\ Systems, University of Technology, Sydney, PO Box 123, Broadway, NSW 2007, Australia
}

\begin{abstract}
In this paper, we develop a fuzzy tri-level decision making (FTLDM) model to deal with decentralized decision making problems with three levels of decision makers. Based on the $\lambda$-cut of fuzzy set, we transform an FTLDM problem into a multiobjective tri-level decision making problem. Based on the linear tri-level $K$ th-best algorithm, the global optimal solution can be obtained. A case study for third-party logistics decision making in supply chain is utilized to illustrate the effectiveness of the proposed algorithm.
\end{abstract}

Keywords: Fuzzy tri-level decision making, $K$ thbest algorithm, Supply chain

\section{Introduction}

Multi-level decision making models, initiated by Von Stackelberg [1], are used to character decentralized decision making problems where decision makers are in a hierarchical organization. Many real-world decision making problem, such as decentralized resource planning [2], highway pricing [3], electronic power market [4] and logistics planning [5] can be formulated as multi-level decision making models. In the last decades, multi-level decision making problems have received more and more attentions [6-8].

Most of the research on multi-level decision making has focused on the bi-level version. In a bi-level decision making model, decision makers in the top level are called the leader and decision makers in the bottom level are called the follower. Decision makers in each level try to optimize their objective functions with partially or without considering the objective of the other level, but the final decision of each level will affect the objective of the other level [9]. In order to get a global optimal solution, a lot of algorithms have been developed, such as the $K$ th-best algorithm [10], the branch and bound algorithm [11], the Kuhn-Tucker condition-based algorithm [12] and heuristics algorithms [13]. However, many decision making problems sometimes involve decision entities at three levels instead of two levels, i.e. the top level, the middle level and the bottom level. For instance, when making a decision in a university, the objectives of the university, the faculty and the department need to be considered, but the three decision entities are in a hierarchical organization. Each decision entity independently optimizes his/her objective, but is affected by the decision of the other two decision entity through external influences [14].

For decision making problems within a tri-level hierarchical organization, Bard and Falk [15] first proposed the necessary conditions for tri-level decision making (TLDM) problems based on Stackelberg game theory and then developed rational reaction sets for each of the decision entities and a cutting-plane algorithm to solve the TLDM problems. Followed by their research, some other studies, such as the penalty-function approach [16], the satisfactory solution-based approach [9] and interactive balance space approach [17], are developed to deal with TLDM problems. In a recent work, Zhang et al. [14] presented a general tri-level decision making model, defined the solution concept of the model and developed a $K$ th-best algorithm to solve the TLDM model. Considering that multiple decision entities may be involved in the middle level and the bottom level for a tri-level decision making problem, Lu et al. [18] proposed a multi-follower tri-level (MFTL) decision making framework, in which 64 standard MFTL decision situations and their possible combinations are identified. The previous studies have significantly advanced the field of tri-level decision making. However, in most situations the coefficients of the optimization problems are often imprecisely known to the decision makers and can't be provided by precise values [19]. For such situations, it may be more appropriate to denote the coefficients of the optimization models with the use of fuzzy sets [20] and optimization problems with fuzzy coefficients have received much attentions in the last few decades [21-24]. The fuzzy coefficients may also appear in a TLDM problem, therefore, there is a need to develop efficient algorithms to deal with the fuzzy tri-level decision making (FTLDM) problems.

In order to do so, the rest of this paper is organized as follows. In section 2, some preliminaries related to fuzzy sets are reviewed. Afterwards, section 3 develops a fuzzy tri-level decision making model and proposes a $K$ th-best algorithm to solve the model based on the $\lambda$-cut of fuzzy sets. In sec- 
tion 4 , a case study of third-party logistics decision making is utilized to illustrate the proposed model and algorithm. Finally, some conclusions are provided in section 5 .

\section{Preliminaries}

Let $R$ denote the set of all real numbers and $R^{n}$ be $n$-dimensional Euclidean space, and the following definitions are given.

Definition 1. [25] A fuzzy number $\tilde{a}$ is defined as a fuzzy set on $R$, whose membership function $\mu_{\tilde{a}}$ satisfies:

(1) $\mu_{\tilde{a}}$ is a mapping from $R$ to the closed interval $[0,1]$

(2) It is normal if there exists $x \in R$ such that $\mu_{\tilde{a}}(x)=1$

(3) For any $\lambda \in[0,1]$, the $\lambda$-cut of $\tilde{a}$ is defined as $a_{\lambda}=\left\{x: \mu_{\tilde{a}}(x) \geq \lambda\right\}$, which is a closed interval, denoted by $\left[a_{\lambda}^{L}, a_{\lambda}^{R}\right]$.

Let $F(R)$ be the set of all fuzzy numbers. By the decomposition theorem of fuzzy set, we have $\tilde{a}=\bigcup_{\lambda \in[0,1]} \lambda\left[a_{\lambda}^{L}, a_{\lambda}^{R}\right]$, for every $\tilde{a} \in F(R)$. A fuzzy number $\tilde{a}$ is called finite if its $\lambda$-cut is a closed interval when $\lambda=0$. Let $F^{*}(R)$ denote the set of all finite fuzzy numbers on $R$.

For any $\tilde{a}, \tilde{b} \in F(R)$ and $\alpha \geq 0$, we have [25]

(1) $\tilde{a}+\tilde{b}=\bigcup_{\lambda \in[0,1]} \lambda\left[a_{\lambda}^{L}+b_{\lambda}^{L}, a_{\lambda}^{R}+b_{\lambda}^{R}\right]$,

(2) $\tilde{a}-\tilde{b}=\bigcup_{\lambda \in[0,1]} \lambda\left[a_{\lambda}^{L}-b_{\lambda}^{R}, a_{\lambda}^{R}-b_{\lambda}^{L}\right]$,

(3) $\alpha \tilde{a}=\bigcup_{\lambda \in[0,1]} \lambda\left[\alpha a_{\lambda}^{L}, \alpha a_{\lambda}^{R}\right]$.

Definition 2. [25] Let $\tilde{a}_{i} \in F(R), i=1,2 \ldots, n$. We define $\tilde{a}=\left(\tilde{a}_{1}, \tilde{a}_{2}, \ldots, \tilde{a}_{n}\right)$

$$
\begin{aligned}
& \mu_{\tilde{a}}: R^{n} \rightarrow[0,1] \\
& x \mapsto \bigwedge_{i=1}^{n} \mu_{\tilde{a}_{i}}\left(x_{i}\right),
\end{aligned}
$$

where $x=\left(x_{1}, x_{2} \ldots, x_{n}\right)^{\mathrm{T}} \in R^{n}$ and $\tilde{a}$ is called an $n$-dimensional fuzzy number on $R^{n}$. If $\tilde{a}_{i} \in F^{*}(R)$, $i=1,2, \ldots, n, \tilde{a}$ is called an $n$-dimensional finite fuzzy number on $R^{n}$.

Let $F\left(R^{n}\right)$ and $F^{*}\left(R^{n}\right)$ be the set of all $n$ dimensional fuzzy numbers and the set of all $n$ dimensional finite fuzzy numbers on $R^{n}$, respectively.

Definition 3. [25] For any $n$-dimensional fuzzy numbers $\tilde{a}, \tilde{b} \in F\left(R^{n}\right)$, we define

(1) $\tilde{a} \succeq \tilde{b}$ iff $a_{i \lambda}^{L} \geqq b_{i \lambda}^{L}$ and $a_{i \lambda}^{R} \geqq b_{i \lambda}^{R}, i=1,2 \ldots, n$, $\lambda \in[0, \overline{1]}$

(2) $\tilde{a} \succeq \tilde{b}$ iff $a_{i \lambda}^{L} \geq b_{i \lambda}^{L}$ and $a_{i \lambda}^{R} \geq b_{i \lambda}^{R}, i=$ $1,2 \ldots, n, \lambda \in[0,1]$

(3) $\tilde{a} \succ \tilde{b}$ iff $a_{i \lambda}^{L}>b_{i \lambda}^{L}$ and $a_{i \lambda}^{R}>b_{i \lambda}^{R}, i=$ $1,2 \ldots, n, \lambda \in[0,1]$.

We call $\succeq, \succeq$ and $\succ$ a fuzzy max order, a stric$\mathrm{t}$ fuzzy max order and a strong fuzzy max order, respectively.

\section{The proposed algorithm}

Let's consider the following fuzzy tri-level decision making (FTLDM) problem:

For $x \in X \subset R^{n}, y \in Y \subset R^{m}, z \in Z \subset R^{p}$, $f_{i}: X \times Y \times Z \rightarrow F^{*}(R), i=1,2,3$,

$$
\begin{array}{cl}
\min _{x \in X} & f_{1}(x, y, z)=\tilde{c}_{1} x+\tilde{d}_{1} y+\tilde{e}_{1} z \\
\text { s.t. } & \tilde{A}_{1} x+\tilde{B}_{1} y+\tilde{C}_{1} z \leq \tilde{b}_{1} \\
\min _{y \in Y} f_{2}(x, y, z)=\tilde{c}_{2} x+\tilde{d}_{2} y+\tilde{e}_{2} z \\
\text { s.t. } \tilde{A}_{2} x+\tilde{B}_{2} y+\tilde{C}_{2} z \leq \tilde{b}_{2} \\
\min _{z \in Z} f_{3}(x, y, z)=\tilde{c}_{3} x+\tilde{d}_{3} y+\tilde{e}_{3} z \\
\text { s.t. } \tilde{A}_{3} x+\tilde{B}_{3} y+\tilde{C}_{3} z \leq \tilde{b}_{3},
\end{array}
$$

where $\tilde{c}_{i} \in F^{*}\left(R^{n}\right), \tilde{d}_{i} \in F^{*}\left(R^{m}\right), \tilde{e}_{i} \in F^{*}\left(R^{p}\right)$, $\tilde{b}_{i} \in F^{*}\left(R^{q_{i}}\right), \tilde{A}_{i}=\left(\tilde{a}_{j k, i}\right)_{q_{i} \times n}, \tilde{a}_{j k, i} \in F^{*}(R)$, $\tilde{B}_{i}=\left(\tilde{b}_{j k, i}\right)_{q_{i} \times m}, \tilde{b}_{j k, i} \in F^{*}(R), \tilde{C}_{i}=\left(\tilde{c}_{j k, i}\right)_{q_{i} \times p}$, $\tilde{c}_{j k, i} \in F^{*}(R), i=1,2,3$. In the FTLDM problem (2), $x, y, z$ denote the decision variables of the top-level, middle-level and bottom-level, respectively, and $f_{1}(x, y, z), f_{2}(x, y, z)$ and $f_{3}(x, y, z)$ denote the objective functions of the top-level, middle-level and bottom-level, respectively.

Associated with the FTLDM problem, we consider the following multi-objective tri-level decision making (MOTLDM) problem:

For $x \in X \subset R^{n}, y \in Y \subset R^{m}, z \in Z \subset R^{p}$, $f_{i}: X \times Y \times Z \rightarrow F^{*}(R), i=1,2,3$,

$$
\begin{aligned}
& \min _{x \in X}\left(f_{1}(x, y, z)\right)_{\lambda}^{L}=c_{1}{ }_{\lambda}^{L} x+d_{1}{ }_{\lambda}^{L} y+e_{1}{ }_{\lambda}^{L} z, \lambda \in[0,1] \\
& \min _{x \in X}\left(f_{1}(x, y, z)\right)_{\lambda}^{R}=c_{1}^{R} x+d_{1}^{R} y+e_{1}^{R} z, \lambda \in[0,1] \\
& \text { s.t. } A_{1}{ }_{\lambda}^{L} x+B_{1}{ }_{\lambda}^{L} y+C_{1}{ }_{\lambda}^{L} z \leq b_{1}{ }_{\lambda}^{L}, \lambda \in[0,1] \\
& A_{1}^{R} x+B_{1}^{R} y+C_{1}^{R} z \leq b_{1}^{R}, \lambda \in[0,1] \\
& \min _{x \in X}\left(f_{2}(x, y, z)\right)_{\lambda}^{L}=c_{2}{ }_{\lambda}^{L} x+d_{2}{ }_{\lambda}^{L} y+e_{2}{ }_{\lambda}^{L} z, \lambda \in[0,1] \\
& \min _{x \in X}\left(f_{2}(x, y, z)\right)_{\lambda}^{R}=c_{2}{ }_{\lambda}^{R} x+d_{2}{ }_{\lambda}^{R} y+e_{2}{ }_{\lambda}^{R} z, \lambda \in[0,1] \\
& \text { s.t. } A_{2}{ }_{\lambda}^{L} x+B_{2}{ }_{\lambda}^{L} y+C_{2}{ }_{\lambda}^{L} z \leq b_{2}^{L}, \lambda \in[0,1] \\
& A_{2}{ }_{\lambda}^{R} x+B_{2}{ }_{\lambda}^{R} y+C_{2}{ }_{\lambda}^{R} z \leq b_{2}{ }_{\lambda}^{R}, \lambda \in[0,1] \\
& \min _{x \in X}\left(f_{3}(x, y, z)\right)_{\lambda}^{L}=c_{3}{ }_{\lambda}^{L} x+d_{3}{ }_{\lambda}^{L} y+e_{3}{ }_{\lambda}^{L} z, \lambda \in[0,1] \\
& \min _{x \in X}\left(f_{3}(x, y, z)\right)_{\lambda}^{R}=c_{3}{ }_{\lambda}^{R} x+d_{3}{ }_{\lambda}^{R} y+e_{3}{ }_{\lambda}^{R} z, \lambda \in[0,1] \\
& \text { s.t. } A_{3}{ }_{\lambda}^{L} x+B_{3}{ }_{\lambda}^{L} y+C_{3}{ }_{\lambda}^{L} z \leq b_{3}{ }_{\lambda}^{L}, \lambda \in[0,1] \\
& A_{3}{ }_{\lambda}^{R} x+B_{3}{ }_{\lambda}^{R} y+C_{3}{ }_{\lambda}^{R} z \leq b_{3}{ }_{\lambda}^{R}, \lambda \in[0,1],
\end{aligned}
$$

where $c_{i \lambda}^{L}, c_{i \lambda}^{R} \in R^{n}, d_{i \lambda}^{L}, d_{i \lambda}^{R} \in R^{m}, e_{i \lambda}^{L}, e_{i \lambda}^{R} \in R^{p}$, $b_{i \lambda}{ }^{L}, b_{i \lambda}^{R} \in R^{q_{i}}, A_{i \lambda}{ }^{L}, A_{i \lambda}^{R} \in R^{q_{i} \times n}, B_{i \lambda}{ }^{L}, B_{i \lambda}^{R} \in$ $R^{q_{i} \times m}, C_{i \lambda}^{L}, C_{i \lambda}^{R} \in R^{q_{i} \times p}, i=1,2,3$.

Theorem 1. Let $\left(x^{*}, y^{*}, z^{*}\right)$ be the solution of the problem (3), then it is also a solution of the FTLDM problem (2).

Proof. The proof is obvious from Definition 3.

Lemma 1. If there is a $\left(x^{*}, y^{*}, z^{*}\right)$ such that $c_{\alpha}^{L} x+$ $d_{\alpha}^{L} y+e_{\alpha}^{L} z \geq c_{\alpha}^{L} x^{*}+d_{\alpha}^{L} y^{*}+e_{\alpha}^{L} z^{*}, c_{\alpha}^{R} x+d_{\alpha}^{R} y+e_{\alpha}^{R} z \geq$ $c_{\alpha}^{R} x^{*}+d_{\alpha}^{R} y^{*}+e_{\alpha}^{R} z^{*}, c_{\beta}^{L} x+d_{\beta}^{L} y+e_{\beta}^{L} z \geq c_{\beta}^{L} x^{*}+d_{\beta}^{L} y^{*}+$ 
$e_{\beta}^{L} z^{*}$, and $c_{\beta}^{R} x+d_{\beta}^{R} y+e_{\beta}^{R} z \geq c_{\beta}^{R} x^{*}+d_{\beta}^{R} y^{*}+e_{\beta}^{R} z^{*}$ for any $(x, y, z)(0 \leq \beta \leq \alpha \leq 1)$, and the coefficients $\tilde{c}$, $\tilde{d}$ and $\tilde{e}$ have trapezoidal fuzzy membership function as

$$
\mu_{\tilde{a}}(t)=\left\{\begin{array}{lr}
0 & t<a_{\beta}^{L} \\
\frac{\alpha-\beta}{a_{\alpha}^{L}-a_{\beta}^{L}}\left(t-a_{\beta}^{L}\right)+\beta & a_{\beta}^{L} \leq t<a_{\alpha}^{L} \\
\alpha & a_{\alpha}^{L} \leq t<a_{\alpha}^{R} \\
\frac{\alpha-\beta}{a_{\alpha}^{R}-a_{\beta}^{R}}\left(t-a_{\beta}^{R}\right)+\beta & a_{\alpha}^{R} \leq t<a_{\beta}^{R} \\
0 & a_{\beta}^{R} \leq t
\end{array},\right.
$$

where $\tilde{a}$ denotes $\tilde{c}, \tilde{d}$ and $\tilde{e}$, respectively, then $c_{\lambda}^{L} x+$ $d_{\lambda}^{L} y+e_{\lambda}^{L} z \geq c_{\lambda}^{L} x^{*}+d_{\lambda}^{L} y^{*}+e_{\lambda}^{L} z^{*}$ and $c_{\lambda}^{R} x+d_{\lambda}^{R} y+$ $e_{\lambda}^{R} z \geq c_{\lambda}^{R} x^{*}+d_{\lambda}^{R} y^{*}+e_{\lambda}^{R} z^{*}, \forall \lambda \in[\beta, \alpha]$.

Proof. By the definition of $\lambda$-cut, we have

$$
\begin{aligned}
& c_{\lambda}^{L}=\frac{c_{\alpha}^{L}-c_{\beta}^{L}}{\alpha-\beta}(\lambda-\beta)+c_{\beta}^{L}, d_{\lambda}^{L}=\frac{d_{\alpha}^{L}-d_{\beta}^{L}}{\alpha-\beta}(\lambda-\beta)+d_{\beta}^{L}, \\
& e_{\lambda}^{L}=\frac{e_{\alpha}^{L}-e_{\beta}^{L}}{\alpha-\beta}(\lambda-\beta)+e_{\beta}^{L} .
\end{aligned}
$$

Thus we have

$$
\begin{aligned}
& c_{\lambda}^{L} x+d_{\lambda}^{L} y+e_{\lambda}^{L} z=\left(\frac{c_{\alpha}^{L}-c_{\beta}^{L}}{\alpha-\beta}(\lambda-\beta)+c_{\beta}^{L}\right) x+\left(\frac{d_{\alpha}^{L}-d_{\beta}^{L}}{\alpha-\beta}\right. \\
& \left.(\lambda-\beta)+d_{\beta}^{L}\right) y+\left(\frac{e_{\alpha}^{L}-e_{\beta}^{L}}{\alpha-\beta}(\lambda-\beta)+e_{\beta}^{L}\right) z \\
& =\frac{\lambda-\beta}{\alpha-\beta}\left(c_{\alpha}^{L} x+d_{\alpha}^{L} y+e_{\alpha}^{L} z\right)+\left(1-\frac{\lambda-\beta}{\alpha-\beta}\right)\left(c_{\beta}^{L} x+d_{\beta}^{L} y+e_{\beta}^{L} z\right) \\
& \geq \frac{\lambda-\beta}{\alpha-\beta}\left(c_{\alpha}^{L} x^{*}+d_{\alpha}^{L} y^{*}+e_{\alpha}^{L} z^{*}\right)+\left(1-\frac{\lambda-\beta}{\alpha-\beta}\right)\left(c_{\beta}^{L} x^{*}+d_{\beta}^{L} y^{*}\right. \\
& \left.+e_{\beta}^{L} z^{*}\right) \\
& =\left(\frac{c_{\alpha}^{L}-c_{\beta}^{L}}{\alpha-\beta}(\lambda-\beta)+c_{\beta}^{L}\right) x^{*}+\left(\frac{d_{\alpha}^{L}-d_{\beta}^{L}}{\alpha-\beta}(\lambda-\beta)+d_{\beta}^{L}\right) y^{*} \\
& +\left(\frac{e_{\alpha}^{L}-e_{\beta}^{L}}{\alpha-\beta}(\lambda-\beta)+e_{\beta}^{L}\right) z^{*} \\
& =c_{\lambda}^{L} x^{*}+d_{\lambda}^{L} y^{*}+e_{\lambda}^{L} z^{*}, \forall \lambda \in[\beta, \alpha] .
\end{aligned}
$$

Similarly, we can prove $c_{\lambda}^{R} x+d_{\lambda}^{R} y+e_{\lambda}^{R} z \geq c_{\lambda}^{R} x^{*}+$ $d_{\lambda}^{R} y^{*}+e_{\lambda}^{R} z^{*}, \forall \lambda \in[\beta, \alpha]$.

Lemma 2. If there is a $\left(x^{*}, y^{*}, z^{*}\right)$ such that $A_{\alpha}^{L} x^{*}+B_{\alpha}^{L} y^{*}+C_{\alpha}^{L} z^{*} \leq b_{\alpha}^{L}, A_{\alpha}^{R} x^{*}+B_{\alpha}^{R} y^{*}+C_{\alpha}^{R} z^{*} \leq$ $b_{\alpha}^{R}, A_{\beta}^{L} x^{*}+B_{\beta}^{L} y^{*}+C_{\beta}^{L} z^{*} \leq b_{\beta}^{L}, A_{\beta}^{R} x^{*}+B_{\beta}^{R} y^{*}+$ $C_{\beta}^{R} z^{*} \leq b_{\beta}^{R}$ for trapezoidal fuzzy number matrices $\tilde{A}, \tilde{B}$ and $\tilde{C}$, then $A_{\lambda}^{L} x^{*}+B_{\lambda}^{L} y^{*}+C_{\lambda}^{L} z^{*} \leq b_{\lambda}^{L}$ and $A_{\lambda}^{R} x^{*}+B_{\lambda}^{R} y^{*}+C_{\lambda}^{R} z^{*} \leq b_{\lambda}^{R}, \forall \lambda \in[\beta, \alpha]$.

Proof. Let $\tilde{A}=\left(\tilde{a}_{i j}\right)_{q \times n}, \quad \tilde{B}=\left(\tilde{b}_{i j}\right)_{q \times m}$, $\tilde{C}=\left(\tilde{c}_{i j}\right)_{q \times p}$ and $\tilde{b}=\left(\tilde{b}_{i}\right)_{q \times 1}$. By the definition of $\lambda$-cut, we have $a_{i j}{ }_{\lambda}^{L}=\frac{a_{i j}^{L}-a_{i j_{\beta}}^{L}}{\alpha-\beta}(\lambda-\beta)+a_{i j}{ }_{\beta}^{L}$, $i=1,2, \ldots, q, \quad j=1,2, \ldots, n ; \quad b_{i j \lambda}{ }_{\lambda}=$ $\frac{b_{i j}{ }_{\alpha}^{L}-b_{i j}{ }_{\beta}^{L}}{\alpha-\beta}(\lambda-\beta)+b_{i j}{ }_{\beta}^{L}, \quad i=1,2, \ldots, q$, $j=1,2, \ldots, m ; c_{i j}{ }_{\lambda}^{L}=\frac{c_{i j}^{L}-c_{i j}^{L}}{\alpha-\beta}(\lambda-\beta)+c_{i j}{ }_{\beta}^{L}$, $i=1,2, \ldots, q, \quad j=1,2, \ldots, p ; \quad b_{i \lambda}{ }^{L}=$
$\frac{b_{i_{\alpha}}^{L}-b_{i_{\beta}}^{L}}{\alpha-\beta}(\lambda-\beta)+b_{i \beta}^{L}, i=1,2, \ldots, q$. Then we have $a_{i j \lambda}{ }_{\lambda} x^{*}+b_{i j}{ }_{\lambda}^{L} y^{*}+a_{i j}{ }_{\lambda}^{L} z^{*}=\left(\frac{a_{i j}{ }_{\alpha}^{L}-a_{i j}{ }_{\beta}^{L}}{\alpha-\beta}(\lambda-\beta)+\right.$ $\left.a_{i j \beta}^{L}\right) x+\left(\frac{b_{i j_{\alpha}}^{L}-b_{i j_{\beta}}^{L}}{\alpha-\beta}(\lambda-\beta)+b_{i j}{ }_{\beta}^{L}\right) y+\left(\frac{c_{i j_{\alpha}}^{L}-c_{i j_{\beta}}^{L}}{\alpha-\beta}\right.$. $\left.(\lambda-\beta)+c_{i j}{ }_{\beta}^{L}\right) z=\frac{\lambda-\beta}{\alpha-\beta}\left(a_{i j}{ }_{\alpha}^{L} x^{*}+b_{i j_{\alpha}}^{L} y^{*}+c_{i j}{ }_{\alpha}^{L} z^{*}\right)$ $+\left(1-\frac{\lambda-\beta}{\alpha-\beta}\right)\left(a_{i j}{ }_{\alpha}^{L} x^{*}+b_{i j}{ }_{\alpha}^{L} y^{*}+c_{i j}{ }_{\alpha}^{L} z^{*}\right) \leq \frac{\lambda-\beta}{\alpha-\beta} b_{i_{\alpha}}^{L}$ $+\left(1-\frac{\lambda-\beta}{\alpha-\beta}\right) b_{i \beta}^{L}=b_{i \lambda}^{L}$.

Thus $A_{\lambda}^{L} x^{*}+B_{\lambda}^{L} y^{*}+C_{\lambda}^{L} z^{*} \leq b_{\lambda}^{L}, \forall \lambda \in[\beta, \alpha]$.

Similarly, we can prove $A_{\lambda}^{R} x^{*}+B_{\lambda}^{R} y^{*}+C_{\lambda}^{R} z^{*} \leq$ $b_{\lambda}^{R}, \forall \lambda \in[\beta, \alpha]$.

Theorem 2. Let $\left(x^{*}, y^{*}, z^{*}\right)$ be the solution of the following MOTLDM decision making problem (5):

$$
\begin{aligned}
& \min _{x \in X}\left(f_{1}(x, y, z)\right)_{\alpha}^{L}=c_{1}{ }_{\alpha}^{L} x+d_{1}{ }_{\alpha}^{L} y+e_{1}{ }_{\alpha}^{L} z \\
& \min _{x \in X}\left(f_{1}(x, y, z)\right)_{\alpha}^{R}=c_{1}{ }_{\alpha}^{R} x+d_{1 \alpha}^{R} y+e_{1 \alpha}^{R} z \\
& \min _{x \in X}\left(f_{1}(x, y, z)\right)_{\beta}^{L}=c_{1}{ }_{\beta}^{L} x+d_{1}{ }_{\beta}^{L} y+e_{1}{ }_{\beta}^{L} z \\
& \min _{x \in X}\left(f_{1}(x, y, z)\right)_{\beta}^{R}=c_{1 \beta}^{R} x+d_{1 \beta}^{R} y+e_{1 \beta}^{R} z \\
& \text { s.t. } A_{1}{ }_{\alpha}^{L} x+B_{1}{ }_{\alpha}^{L} y+C_{1}{ }_{\alpha}^{L} z \leq b_{1}{ }_{\alpha}^{L} \\
& A_{1 \alpha}{ }_{\alpha}^{R} x+B_{1 \alpha}{ }_{\alpha}^{R} y+C_{1}{ }_{\alpha}^{R} z \leq b_{1 \alpha}^{R} \\
& A_{1}{ }_{\beta}^{L} x+B_{1 \beta}^{L} y+C_{1 \beta}^{L} z \leq b_{1 \beta}^{L} \\
& A_{1 \beta}^{R} x+B_{1}{ }_{\beta}^{R} y+C_{1 \beta}^{R} z \leq b_{1}^{R} \\
& \min _{x \in X}\left(f_{2}(x, y, z)\right)_{\alpha}^{L}=c_{2}{ }_{\alpha}^{L} x+d_{2}{ }_{\alpha}^{L} y+e_{2}{ }_{\alpha}^{L} z \\
& \min _{x \in X}\left(f_{2}(x, y, z)\right)_{\alpha}^{R}=c_{2}{ }_{\alpha}^{R} x+d_{2}{ }_{\alpha}^{R} y+e_{2}{ }_{\alpha}^{R} z \\
& \min _{x \in X}\left(f_{2}(x, y, z)\right)_{\beta}^{L}=c_{2}{ }_{\beta}^{L} x+d_{2}{ }_{\beta}^{L} y+e_{2}{ }_{\beta}^{L} z \\
& \min _{x \in X}\left(f_{2}(x, y, z)\right)_{\beta}^{R}=c_{2}{ }_{\beta}^{R} x+d_{2}{ }_{\beta}^{R} y+e_{2}{ }_{\beta}^{R} z \\
& \text { s.t. } A_{2}{ }_{\alpha}^{L} x+B_{2}{ }_{\alpha}^{L} y+C_{2}{ }_{\alpha}^{L} z \leq b_{2}{ }_{\alpha}^{L} \\
& A_{2}{ }_{\alpha}^{R} x+B_{2}{ }_{\alpha}^{R} y+C_{2}{ }_{\alpha}^{R} z \leq b_{2}{ }_{\alpha}^{R} \\
& A_{2}{ }_{\beta}^{L} x+B_{2 \beta}^{L} y+C_{2 \beta}^{L} z \leq b_{2 \beta}^{L} \\
& A_{2}{ }_{\beta}^{R} x+B_{2 \beta}^{R} y+C_{2 \beta}^{R} z \leq b_{2 \beta}^{R} \\
& \min _{x \in X}\left(f_{3}(x, y, z)\right)_{\alpha}^{L}=c_{3}{ }_{\alpha}^{L} x+d_{3}{ }_{\alpha}^{L} y+e_{3}{ }_{\alpha}^{L} z \\
& \min _{x \in X}\left(f_{3}(x, y, z)\right)_{\alpha}^{R}=c_{3}{ }_{\alpha}^{R} x+d_{3}{ }_{\alpha}^{R} y+e_{3}{ }_{\alpha}^{R} z \\
& \min _{x \in X}\left(f_{3}(x, y, z)\right)_{\beta}^{L}=c_{3}{ }_{\beta}^{L} x+d_{3}{ }_{\beta}^{L} y+e_{3}{ }_{\beta}^{L} z \\
& \min _{x \in X}\left(f_{3}(x, y, z)\right)_{\beta}^{R}=c_{3}{ }_{\beta}^{R} x+d_{3}{ }_{\beta}^{R} y+e_{3}^{R} z \\
& \text { s.t. } A_{3}{ }_{\alpha}^{L} x+B_{3}{ }_{\alpha}^{L} y+C_{3}{ }_{\alpha}^{L} z \leq b_{3}{ }_{\alpha}^{L} \\
& A_{3 \alpha}{ }^{R} x+B_{3 \alpha}{ }_{\alpha}^{R} y+C_{3 \alpha}{ }_{\alpha}^{R} z \leq b_{3 \alpha}^{R} \\
& A_{3}{ }_{\beta}^{L} x+B_{3}{ }_{\beta}^{L} y+C_{3}{ }_{\beta}^{L} z \leq b_{3}{ }_{\beta}^{L} \\
& A_{3 \beta}^{R} x+B_{3 \beta}^{R} y+C_{3 \beta}^{R} z \leq b_{3 \beta}^{R} \text {. }
\end{aligned}
$$

If the coefficients of the FTLDM problem (2) have trapezoidal membership functions as Eq. (4), the 
solution is also the solution of the FTLDM problem (2).

Proof. By Lemma 1, if $\left(x^{*}, y^{*}, z^{*}\right)$ satisfies

$$
\begin{aligned}
& \min _{x \in X}\left(f_{1}(x, y, z)\right)_{\alpha}^{L}=c_{1}{ }_{\alpha}^{L} x^{*}+d_{1}{ }_{\alpha}^{L} y^{*}+e_{1}{ }_{\alpha}^{L} z^{*}, \\
& \min _{x \in X}\left(f_{1}(x, y, z)\right)_{\alpha}^{R}=c_{1}{ }_{\alpha}^{R} x^{*}+d_{1}{ }_{\alpha}^{R} y^{*}+e_{1}{ }_{\alpha}^{R} z^{*}, \\
& \min _{x \in X}\left(f_{1}(x, y, z)\right)_{\beta}^{L}=c_{1}{ }_{\beta}^{L} x^{*}+d_{1}{ }_{\beta}^{L} y^{*}+e_{1}^{L} z^{*}, \\
& \min _{x \in X}\left(f_{1}(x, y, z)\right)_{\beta}^{R}=c_{1 \beta}^{R} x^{*}+d_{1 \beta}^{R} y^{*}+e_{1}^{R} z^{*},
\end{aligned}
$$

then we have

$$
\begin{aligned}
& \min _{x \in X}\left(f_{1}(x, y, z)\right)_{\lambda}^{L}=c_{1 \lambda}^{L} x^{*}+d_{1 \lambda}^{L} y^{*}+e_{1 \lambda}^{L} z^{*}, \\
& \min _{x \in X}\left(f_{1}(x, y, z)\right)_{\lambda}^{R}=c_{1 \lambda}^{R} x^{*}+d_{1 \lambda}^{R} y^{*}+e_{1 \lambda}^{R} z^{*}, \quad \lambda \in[\beta, \alpha] .
\end{aligned}
$$

Similarly, we have

$$
\begin{aligned}
& \min _{y \in Y}\left(f_{2}(x, y, z)\right)_{\lambda}^{L}=c_{2 \lambda}^{L} x^{*}+d_{2 \lambda}^{L} y^{*}+e_{2 \lambda}^{L} z^{*}, \\
& \min _{y \in Y}\left(f_{2}(x, y, z)\right)_{\lambda}^{R}=c_{2 \lambda}^{R} x^{*}+d_{2 \lambda}^{R} y^{*}+e_{2 \lambda}^{R} z^{*}, \lambda \in[\beta, \alpha] ; \\
& \min _{z \in Z}\left(f_{3}(x, y, z)\right)_{\lambda}^{L}=c_{3 \lambda}^{L} x^{*}+d_{3 \lambda}^{L} y^{*}+e_{3 \lambda}^{L} z^{*}, \\
& \min _{z \in Z}\left(f_{3}(x, y, z)\right)_{\lambda}^{R}=c_{3 \lambda}^{R} x^{*}+d_{3 \lambda}^{R} y^{*}+e_{3 \lambda}^{R} z^{*}, \lambda \in[\beta, \alpha] .
\end{aligned}
$$

By Lemma 2, if $\left(x^{*}, y^{*}, z^{*}\right)$ satisfies

$$
\begin{aligned}
& A_{i_{\alpha}}{ }^{L} x+B_{i_{\alpha}}{ }^{L} y+C_{i_{\alpha}}{ }^{L} z \leq b_{i \alpha}{ }_{\alpha}^{L}, \\
& A_{i_{\alpha}}^{R} x+B_{i_{\alpha}}^{R} y+C_{i_{\alpha}}^{R} z \leq b_{i_{\alpha}}^{R}, \\
& A_{i_{\beta}}{ }^{R} x+B_{i \beta}{ }_{i}^{L} y+C_{i \beta}{ }^{L} z \leq b_{i \beta}{ }_{i \beta}^{L}, \\
& A_{i \beta}^{R} x+B_{i \beta}^{R} y+C_{i \beta}^{R} z \leq b_{i \beta}^{R}, i=1,2,3,
\end{aligned}
$$

then

$$
A_{i \lambda}^{L} x^{*}+B_{i \lambda}^{L} y^{*}+C_{i \lambda}^{L} z^{*} \leq b_{i \lambda}^{L}, \text { and } A_{i \lambda}^{R} x^{*}+B_{i \lambda}^{R} y^{*}+
$$
$C_{i \lambda}^{R} z^{*} \leq b_{i \lambda}^{R}$ will hold, $\forall \lambda \in[\beta, \alpha], i=1,2,3$.

To summarize, the proof of Theorem 2 is completed.

By Theorem 2, solving the FTLDM problem (2) is equivalent to solving the MOTLDM problem (5). In order to solve the problem (5), the linear weighted method can be used. Therefore, the problem (5) can be transformed into the problem (6).

The problem (6) is a TLDM problem. According to the theorem provided by [14], the solution to a TLDM problem occurs at a vertex of the constraint region, thus the linear tri-level $K$ th-best algorithm [14] can be used to solve the problem.

Let $S=\left\{(x, y, z): x \in X, y \in Y, z \in Z, A_{i_{\alpha}}{ }^{L} x+\right.$ $B_{i_{\alpha}}{ }^{L} y+C_{i \alpha}{ }^{L} z \leq b_{i \alpha}{ }^{L}, A_{i_{\alpha}}^{R} x+B_{i_{\alpha}}^{R} y+C_{i_{\alpha}}^{R} z \leq$ $b_{i \alpha}^{R}, \quad A_{i \beta}^{L} x+B_{i \beta}{ }^{L} y+C_{i \beta}^{L} z \leq b_{i \beta}^{L}, \quad A_{i \beta}^{R} x+B_{i \beta}^{R} y+$ $\left.C_{i \beta}^{R} z \leq b_{i \beta}^{R}, i=1,2,3\right\}, S(x)=\{(y, z) \in Y \times Z$ : $A_{i}{ }_{i \alpha}^{L} x+B_{i \alpha}{ }_{i \alpha} y+C_{i}{ }_{i \alpha}^{L} z \leq b_{i}{ }_{i \alpha}^{L}, A_{i_{\alpha}}{ }^{R} x+B_{i_{\alpha}}{ }^{R} y+C_{i_{\alpha}}^{R} z \leq$ $b_{i \alpha}^{R}, \quad A_{i \beta}^{L} x+B_{i \beta}^{L} y+C_{i \beta}^{L} z \leq b_{i \beta}^{L}, A_{i \beta}^{R} x+B_{i \beta}^{R} y+$ $\left.C_{i \beta}^{R} z \leq b_{i \beta}^{R}, i=2,3\right\}, S(x, y)=\left\{z \in Z: A_{3}{ }_{\alpha}^{L} x+\right.$

$$
\begin{aligned}
& \min _{x \in X} g_{1}(x, y, z)=\left(c_{1}^{L}+c_{1}^{R}+c_{1}^{L}+c_{1}^{L}\right) x+\left(d_{1}^{L}+d_{1}^{L}{ }_{\alpha}^{R}\right. \\
& \left.\left.+d_{1}^{L}+d_{1}^{R}\right) y+e_{1}^{L}+e_{1}^{R}+e_{1}^{L}+e_{1}^{R}\right) z \\
& \text { s.t. } A_{1}{ }_{\alpha}^{L} x+B_{1}{ }_{\alpha}^{L} y+C_{1}{ }_{\alpha}^{L} z \leq b_{1}{ }_{\alpha}^{L} \\
& A_{1}{ }_{\alpha}^{R} x+B_{1}{ }_{\alpha}^{R} y+C_{1}{ }_{\alpha}^{R} z \leq b_{1}{ }_{\alpha}^{R} \\
& A_{1}{ }_{\beta}^{L} x+B_{1}^{L}{ }_{\beta}^{L} y+C_{1}{ }_{\beta}^{L} z \leq b_{1}^{L} \\
& A_{1}{ }_{\beta}^{R} x+B_{1}^{R} y+C_{1}^{R} z \leq b_{1}^{R} \\
& \min _{y \in Y} g_{2}(x, y, z)=\left(c_{2}{ }_{\alpha}^{L}+c_{2} \underset{\alpha}{R}+c_{2}{ }_{\beta}^{L}+c_{2}{ }_{\beta}^{R}\right) x+\left(d_{2}{ }_{\alpha}^{L}+\right. \\
& \left.d_{2}^{R}+d_{2}^{L}{ }_{\beta}^{L}+d_{2}^{R}\right) y+\left(e_{2}^{L}+e_{2}^{R}+e_{2}^{L}{ }_{\beta}^{L}+e_{2}^{R}\right) z \\
& \text { s.t. } A_{2}{ }_{\alpha}^{L} x+B_{2}{ }_{\alpha}^{L} y+C_{2}{ }_{\alpha}^{L} z \leq b_{2}{ }_{\alpha}^{L} \\
& A_{2}{ }_{\alpha}^{R} x+B_{2}{ }_{\alpha}^{R} y+C_{2}{ }_{\alpha}^{R} z \leq b_{2}{ }_{\alpha}^{R} \\
& A_{2}{ }_{\beta}^{L} x+B_{2}{ }_{\beta}^{L} y+C_{2}{ }_{\beta}^{L} z \leq b_{2}^{L} \\
& A_{2}{ }_{\beta}^{R} x+B_{2}{ }_{\beta}^{R} y+C_{2}{ }_{\beta}^{R} z \leq b_{2}{ }_{\beta}^{R} \\
& \min _{z \in Z} g_{3}(x, y, z)=\left(c_{3}^{L}+c_{3}{ }_{\alpha}^{R}+c_{3}{ }_{\beta}^{L}+c_{3}{ }_{\beta}^{R}\right) x+\left(d_{3}{ }_{\alpha}^{L}\right. \\
& \left.+d_{3 \alpha}^{R}+d_{3}^{L}+d_{3}^{R}\right) y+\left(e_{3}^{L}+e_{3 \alpha}^{R}+e_{3}^{L}+e_{3}^{R}\right) z \\
& \text { s.t. } A_{3}{ }_{\alpha}^{L} x+B_{3}{ }_{\alpha}^{L} y+C_{3}{ }_{\alpha}^{L} z \leq b_{3}{ }_{\alpha}^{L} \\
& A_{3}{ }_{\alpha}^{R} x+B_{3}{ }_{\alpha}^{R} y+C_{3}{ }_{\alpha}^{R} z \leq b_{3}{ }_{\alpha}^{R} \\
& A_{3}{ }_{\beta}^{L} x+B_{3}{ }_{\beta}^{L} y+C_{3}{ }_{\beta}^{L} z \leq b_{3}{ }_{\beta}^{L} \\
& A_{3}{ }_{\beta}^{R} x+B_{3}{ }_{\beta}^{R} y+C_{3}{ }_{\beta}^{R} z \leq b_{3}^{R} \text {. }
\end{aligned}
$$

$B_{3 \alpha}^{L} y+C_{3}{ }_{\alpha}^{L} z \leq b_{3 \alpha}^{L}, \quad \overline{A_{3} \alpha_{\alpha}^{R} x+B_{3}{ }_{\alpha}^{R} y+C_{3 \alpha}^{R} z \leq}$ $b_{3 \alpha}^{R}, \quad A_{3 \beta}^{L} x+B_{3 \beta}^{L} y+C_{3 \beta}^{L} z \leq b_{3 \beta}^{L}, \quad A_{3 \beta}^{R} x+$ $\left.B_{3 \beta}^{R} y+C_{3 \beta}^{R} z \leq b_{3 \beta}^{R}\right\}$ and $P(x)=\{(y, z)$ : $(y, z) \in \arg \min \left\{g_{2}(x, \hat{y}, \hat{z}):(\hat{y}, \hat{z}) \in S(x), \hat{z} \in\right.$ $\left.\left.\arg \min \left\{g_{3}(x, \hat{y}, \tilde{z}): \tilde{z} \in S(x, \hat{y})\right\}\right\}\right\}$, then the algorithm can be described as follows:

\section{[Algorithm 1]}

Step 1: Transform the FTLDM problem (2) into problem (6).

Step 2: Set $i \leftarrow 1$. Solve the linear programming problem $\min \left\{g_{1}(x, y, z):(x, y, z) \in S\right\}$ by the simplex method to obtain the optimal solution $\left(x_{[1]}, y_{[1]}, z_{[1]}\right)$, and then let $W=$ $\left\{\left(x_{[1]}, y_{[1]}, z_{[1]}\right)\right\}$ and $T=\phi$.

Step 3: Let $x=x_{[i]}$ and utilize the bi-level $K$ thbest algorithm [10] to solve the bi-level decision making problem

$$
\min \left\{g_{2}(x, y, z):(y, z) \in P\left(x_{[i]}\right)\right\}
$$

Let $(\hat{y}, \hat{z})$ be the optimal solution to $(7)$. If $\hat{y}=y_{[i]}$ and $\hat{z}=z_{[i]},\left(x_{[i]}, y_{[i]}, z_{[i]}\right)$ is the global optimum of the FTLDM problem (2) and $K^{*}=i$, go to Step 6; otherwise, go to Step 4.

Step 4: Let $W_{[i]}$ denote the set of adjacent extreme points of $\left(x_{[i]}, y_{[i]}, z_{[i]}\right)$ such that $g_{1}(x, y, z) \geq g_{1}\left(x_{[i]}, y_{[i]}, z_{[i]}\right)$. Let $T=$ $T \cup\left\{\left(x_{[i]}, y_{[i]}, z_{[i]}\right)\right\}$ and $W=\left(W \cup W_{[i]}\right) \backslash T$. Go to Step 5 . 
Step 5: Set $i \leftarrow i+1$ and choose $\left\{\left(x_{[i]}, y_{[i]}, z_{[i]}\right)\right\}$ from $W$ such that $g_{1}\left(x_{[i]}, y_{[i]}, z_{[i]}\right)=$ $\min \left\{g_{1}(x, y, z):(x, y, z) \in W\right\}$. Go to Step 3.

Step 6: Output the solution of the FTLDM problem (2). [End]

\section{A case study on third-party logistics decision making}

For the purpose of this research, a case study on third-party logistics decision making is developed based on the FTLDM model. Nowadays companies are focusing on enhancing their competitive advantage in order to meet the economy globalization. One effective way is developing their core businesses in the supply chains, while outsourcing non-core businesses, among which logistics is the main operation to be outsourced [26]. Especially with the rapid development of the Internet of Things techniques, companies can grasp the information of the products in real time online and more and more companies have begun to outsource their main logistics operation to third-party logistics (3PL) companies in recent years. In this case, the vendor, the logistics company and the distributor form a hierarchical organization. The three decision entities have their own objectives, for instance, maximize the profits or minimize the production costs. In addition, each decision entity has its own constraints and variables. When making a decision in the supply chain, the logistics company fully considers the decision of the vendor and the distributor also considers the decision of the logistics company. At the same time, the vendor also takes into account the reaction of the logistics company and the logistics company likewise considers the reaction of the distributor. Due to the uncertainty of judgments, the coefficients of the three entities cannot be expressed with exact numerical numbers. For instance, the unit profit of a product may be "about $\$ 10$ ". Therefore the decision making problem faced to the decision entities is usually with fuzzy coefficients. In order to make an optimal decision, the FTLDM models can be utilized.

In a supply chain, a high-tech product vendor wants to minimize its production cost (represented by $\min f_{1}(x, y, z)$ ) and $x$ (the number of orders on the planning horizon) is the decision variable. The logistics company needs to determine the number of trucks to be rent for the distribution $(y)$, with the objective of minimizing the overall transportation costs $\left(\min f_{2}(x, y, z)\right)$. The distributor has the objective "maximizing the overall profit" $\left(\max f_{3}(x, y, z)\right)$ and $z$ (the order quantity per lot-size) is its decision variable. The objectives at the three levels are subject to their particular constraints, for instance, the cost of inventory and the supply of materials. We simplify the fuzzy tri-level decision making problem of the supply chain as follows:

$$
\begin{array}{ll}
\min _{x \in X} & f_{1}(x, y, z)=\tilde{1} x-\tilde{4} y+\tilde{2} z+\tilde{2} \\
\text { s.t. } & -\tilde{1} x+\tilde{3} y+\tilde{1} z \leq \tilde{4} \\
& \min _{y \in Y} f_{2}(x, y, z)=\tilde{1} x+\tilde{1} y-\tilde{1} z+\tilde{2} \\
\text { s.t. } \tilde{1} x-\tilde{1} y-\tilde{2} z \leq \tilde{0} \\
\quad \max _{z \in Z} f_{3}(x, y, z)=-\tilde{1} x+\tilde{2} y+\tilde{1} z+\tilde{3} \\
\text { s.t. }-\tilde{1} x-\tilde{1} y+\tilde{3} z \leq \tilde{0},
\end{array}
$$

where

$$
\begin{aligned}
& \mu_{\tilde{0}}(t)=\left\{\begin{array}{lr}
0 & t<-1 \\
t+1 & -1 \leq t<0 \\
1-t & 0 \leq t<1 \\
0 & 1 \leq t
\end{array},\right. \\
& \mu_{\tilde{1}}(t)=\left\{\begin{array}{lr}
0 & t<0 \\
t & 0 \leq t<1 \\
2-t & 1 \leq t<2 \\
0 & 2 \leq t
\end{array},\right. \\
& \mu_{\tilde{2}}(t)=\left\{\begin{array}{lr}
0 & t<1 \\
t-1 & 1 \leq t<2 \\
3-t & 2 \leq t<3 \\
0 & 3 \leq t
\end{array},\right. \\
& \mu_{\tilde{3}}(t)=\left\{\begin{array}{lr}
0 & t<2 \\
t-2 & 2 \leq t<3 \\
4-t & 3 \leq t<4 \\
0 & 4 \leq t
\end{array},\right. \\
& \mu_{\tilde{4}}(t)=\left\{\begin{array}{lr}
0 & t<3 \\
t-3 & 3 \leq t<4 \\
5-t & 4 \leq t<5 \\
0 & 5 \leq t
\end{array} .\right.
\end{aligned}
$$

Now, we present a detailed procedure to solve the above fuzzy tri-level decision making problem in supply chain.

Step 1: We transform the problem into

$$
\begin{aligned}
& \min _{x \in X} g_{1}(x, y, z)=(0+2+1) x+(-5-3-4) y \\
& +(1+3+2) z+(1+3+2)=3 x-12 y+6 z+6 \\
& \text { s.t. }-2 x+2 y+0 z \leq 3,0 x+4 y+2 z \leq 5 \\
& \quad-1 x+3 y+1 z \leq 4 \\
& \quad \min _{y \in Y} g_{2}(x, y, z)=(0+2+1) x+(0+2+1) y \\
& +(-2+0-1) z+(1+3+2)=3 x+3 y-3 z+6 \\
& \text { s.t. } 0 x-2 y-3 z \leq-1,2 x-0 y-1 z \leq 1 \\
& \quad 1 x-y-2 z \leq 0 \\
& \quad \max _{z \in Z} g_{3}(x, y, z)=(-2+0-1) x+(1+3+2) y \\
& \quad+(0+2+1) z+(2+4+3)=-3 x+6 y+3 z+9 \\
& \quad \text { s.t. } \quad-2 x-2 y+2 z \leq-1,0 x+0 y+4 z \leq 1 \\
& \quad-x-y+3 z \leq 0 .
\end{aligned}
$$


Step 2: Let $i=1$, solve the following linear programming problem (10) using the simplex method:

$$
\begin{array}{ll}
\min _{x \in X} & g_{1}(x, y, z)=3 x-12 y+6 z+6 \\
\text { s.t. } & -2 x+2 y+0 z \leq 3,0 x+4 y+2 z \leq 5 \\
& -1 x+3 y+1 z \leq 4,0 x-2 y-3 z \leq-1 \\
& 2 x-0 y-1 z \leq 1,1 x-y-2 z \leq 0 \\
& -2 x-2 y+2 z \leq-1,0 x+0 y+4 z \leq 1 \\
& -x-y+3 z \leq 0 .
\end{array}
$$

The solution to the problem (10) is $\left(x_{[1]}, y_{[1]}, z_{[1]}\right)=(0,1.25,0)$. Let $W=\{(0,1.25,0)\}$ and $T=\phi$.

Step 3: Let $x=0$, utilize the bounded simplex method to solve the following model:

$$
\begin{aligned}
\min _{y \in Y} & g_{2}(x, y, z)=3 x+3 y-3 z+6 \\
\text { s.t. } & -2 x+2 y+0 z \leq 3,0 x+4 y+2 z \leq 5 \\
& -1 x+3 y+1 z \leq 4,0 x-2 y-3 z \leq-1 \\
& 2 x-0 y-1 z \leq 1,1 x-y-2 z \leq 0 \\
& -2 x-2 y+2 z \leq-1,0 x+0 y+4 z \leq 1 \\
& -x-y+3 z \leq 0, x=0 .
\end{aligned}
$$

The solution is $\left(y_{[1]}^{\prime}, z_{[1]}^{\prime}\right)=(0.5,0)$.

Let $x=0, y=0.5$, utilize the bounded simplex method to solve the following model:

$$
\begin{aligned}
\max _{z \in Z} & g_{3}(x, y, z)=-3 x+6 y+3 z+9 \\
\text { s.t. } & -2 x+2 y+0 z \leq 3,0 x+4 y+2 z \leq 5 \\
& -1 x+3 y+1 z \leq 4,0 x-2 y-3 z \leq-1 \\
& 2 x-0 y-1 z \leq 1,1 x-y-2 z \leq 0 \\
& -2 x-2 y+2 z \leq-1,0 x+0 y+4 z \leq 1 \\
& -x-y+3 z \leq 0 \\
& x=0, y=0.5 .
\end{aligned}
$$

The solution is $z_{[1]}^{\prime \prime}=0$. Since $z_{[1]}^{\prime \prime}=z_{[1]}^{\prime}$ and $y_{[1]}^{\prime} \neq y_{[1]}$, go to Step 4 .

Step 4: The set of the adjacent extreme points of $\left(x_{[1]}, y_{[1]}, z_{[1]}\right)$ is $W_{[1]}=$ $\{(0,0.5,0),(0.5,1.25,0),(0,1.125,0.25)\}, \quad$ thus we have $T=\{(0,1.25,0)\}$ and $W=$ $\{(0,0.5,0),(0.5,1.25,0),(0,1.125,0.25)\}$.

Step 5: Update $i=2$, choose $\left(x_{[i]}, y_{[i]}, z_{[i]}\right)=$ $(0.5,1.25,0)$ and go to Step 3. The algorithm stops after 8 iterations, i.e. $K^{*}=8$. Details of the algorithm implementation are omitted.

Step 6: The optimal solution to the fuzzy trilevel decision making problem (8) is $\left(x^{*}, y^{*}, z^{*}\right)=$ $(0.625,0.125,0.25)$. The objective function values of the three levels are as follows: $\tilde{f}_{1}^{*}=\tilde{1} x^{*}-\tilde{4} y^{*}+\tilde{2} z^{*}+$ $\tilde{2}, \tilde{f}_{2}^{*}=\tilde{1} x^{*}+\tilde{1} y^{*}-\tilde{1} z^{*}+\tilde{2}, \tilde{f}_{3}^{*}=-\tilde{1} x^{*}+\tilde{2} y^{*}+\tilde{1} z^{*}+\tilde{3}$.

The results shows that when $x=0.625, y=0.125$ and $z=0.25$, the vendor can obtain the minimized production cost, the logistics company can have a minimized transportation cost and the distributor can get the maximized profit.

\section{Conclusions}

Many organization decision making problems can be formulated as tri-level decision making models. When formulating a tri-level decision making model, the coefficients of objective functions and constraints are sometimes in the form of fuzzy numbers. In this paper, we first present a tri-level decision making model with fuzzy parameters and transfor$\mathrm{m}$ the model into a multi-objective tri-level decision making problem based on the $\lambda$-cut, which can be solved by the tri-level $K$ th-best algorithm.

In terms of future research, we will consider fuzzy tri-level decision making problems with multiple objectives and multiple followers. A web-based fuzzy tri-level decision support system will be also developed to support the third-party logistics decision making.

\section{Acknowledgements}

This work was partly supported by the National Natural Science Foundation of China under grant 71171030 and Australian Research Council (ARC) under discovery grant DP110103733.

\section{References}

[1] H Von Stackelberg. Theory of the Market Economy. Oxford University Press, Oxford, 1952.

[2] Z. Lukac, K. Soric, and V. V. Rosenzweig. Production planning problem with sequence dependent setups as a bilevel programming problem. European Journal of Operational Research, 187(3):1504-1512, 2008.

[3] M. Labbé, P. Marcotte, and G. Savard. A bilevel model of taxation and its application to optimal highway pricing. Management Science, 44(12-Part-1):1608-1622, 1998.

[4] G. Zhang, G. Zhang, Y. Gao, and J. Lu. Competitive strategic bidding optimization in electricity markets using bilevel programming and swarm technique. IEEE Transactions on Industrial Electronics, 58(6):2138-2146, 2011.

[5] G. Zhang and J. Lu. Model and approach of fuzzy bilevel decision making for logistics planning problem. Journal of Enterprise Information Management, 20(2):178-197, 2007.

[6] J. F. Bard. Practical Bilevel Optimization: Algorithms and Applications. Kluwer Academic Publishers, Boston, MA, 1998.

[7] M. Sakawa, I. Nishizaki, and Y. Uemura. Interactive fuzzy programming for multi-level linear programming problems with fuzzy parameters. Fuzzy Sets and Systems, 109(1):3-19, 2000.

[8] F. Tiryaki. Interactive compensatory fuzzy programming for decentralized multi-level linear programming (DMLLP) problems. Fuzzy Sets and Systems, 157(23):3072-3090, 2006. 
[9] Y. Lai. Hierarchical optimization: A satisfactory solution. Fuzzy Sets and Systems, 77(3):321335, 1996.

[10] W. F. Bialas and M. H. Karwan. Two-level linear programming. Management Science, 30(8):1004-1020, 1984.

[11] J. Bard and J. Moore. A branch and bound algorithm for the bilevel programming problem. SIAM Journal on Scientific and Statistical Computing, 11(2):281-292, 1990.

[12] J. Lu, C. Shi, G. Zhang, and T. Dillon. Model and extended kuhn-tucker approach for bilevel multi-follower decision making in a referentialuncooperative situation. Journal of Global Optimization, 38(4):597-608, 2007.

[13] Y. Gao, G. Zhang, J. Lu, and H. M. Wee. Particle swarm optimization for bi-level pricing problems in supply chains. Journal of Global Optimization, 51(2):245-254, 2011.

[14] G. Zhang, J. Lu, J. Montero, and Y. Zeng. Model, solution concept, and $K$ th-best algorithm for linear trilevel programming. Information Sciences, 180(4):481-492, 2010.

[15] J Bard and J Falk. Necessary conditions for the linear three-level programming problem. In Proceedings of the 21st IEEE Conference on Decision and Control, volume 21, pages 642 646, 1982.

[16] D. J. White. Penalty function approach to linear trilevel programming. Journal of Optimization Theory and Applications, 93(1):183-197, 1997.

[17] M. A. Abo-Sinna and I. A. Baky. Interactive balance space approach for solving multi-level multi-objective programming problems. Information Sciences, 177(16):3397 - 3410, 2007.

[18] J. Lu, G. Zhang, J. Montero, and L. Garmendia. Multifollower trilevel decision making models and system. IEEE Transactions on Industrial Informatics, 8(4):974-985, 2012.

[19] Y. Gao, G. Zhang, J. Ma, and J. Lu. A $\lambda$-cut and goal-programming-based algorithm for fuzzy-linear multiple-objective bilevel optimization. IEEE Transactions on Fuzzy Systems, 18(1):1-13, 2010.

[20] L. A. Zadeh. Fuzzy sets. Information and Control, 8(3):338-353, 1965.

[21] M Delgado, J Kaprzyk, JL Verdegay, and MA Vila. Fuzzy Optimization: Recent Advances. Physica-Verlag, New York, 1994.

[22] Y. J. Lai and C. L. Hwang. Fuzzy Multiple Objective DecisionMaking - Methods and Applications. Springer, Berlin, 1994.

[23] M. Sakawa. Fuzzy Sets and Interactive Multiobjective Optimization. Plenum, New York, 1993.

[24] Y. C. Liou, S. Y. Wu, and J. C. Yao. Bilevel decision with generalized semi-infinite optimization for fuzzy mappings as lower level problems. Fuzzy Optimization and Decision Making,
4(1):41-50, 2005.

[25] G. Zhang, Y. Wu, M. Remias, and J. Lu. Formulation of fuzzy linear programming problems as four-objective constrained optimization problems. Applied Mathematics and Computation, 139(2-3):383-399, 2003.

[26] F. Li, L. Li, C. Jin, R. Wang, H. Wang, and L. Yang. A 3PL supplier selection model based on fuzzy sets. Computers $\&$ Operations Research, 39(8):1879 - 1884, 2012. 